\title{
A novel evaluation tool for enhancing the selection of natural fibres for polymeric composites based on fiber moisture content criterion
}

\begin{abstract}
A systematic evaluation tool for natural fibers' capabilities based on moisture content criterion (MCC) was developed and introduced as a new evaluation method. This MCC evaluation tool is designed to predict the behavior of the available natural fibers regarding distinctive desirable characteristics under the effect of the moisture absorption phenomenon. Here, the capabilities of different natural fiber types commonly used in industry, in addition to date palm fibers, were systematically investigated based on MCC. The results demonstrated that MCC is capable of predicting the relative reduction of fiber performance regarding a particular beneficial property because of the effect of moisture absorption. The strong agreements between the predicted values of MCC and results reported in the literature verify its usefulness as an evaluation tool and demonstrate its added value steps in predicting the relative behavior of fibers with a minimal range of errors compared with experimental measurements. Therefore, MCC is capable of better evaluating natural fibers regarding distinctive criteria in a systematic manner, leading to more realistic decisions about their capabilities and therefore enhancing the selection process for both better sustainable design possibilities and industrial product development.
\end{abstract}

Keyword: Natural fibers; Moisture content; Polymeric composites; Sustainable design; Evaluation tool; Date palm fibers 